\title{
Abuso Sexual Infantil: percepción social de los y las docentes en primarias en Mérida, Yucatán
}

\section{Child Sexual Abuse: the social perception of elementary school teachers in Mérida, Yucatán}

DOI: https://doi.org/10.32870/dse.v0i23.738

Mayra Canela Parejas*

Johana Verónica Carballido Arjona**

Marian Jazmin Dzul Dzul***

Michel Patricia Herrera Núñez****

Karla Sofía Ortiz Contreras $* * * * *$

\begin{abstract}
Resumen
El Abuso Sexual Infantil (ASI) es un tema de interés a nivel mundial. El tema elegido alude a la percepción social que poseen los y las docentes en escuelas primarias en la ciudad de Mérida acerca del abuso sexual infantil. La presente investigación, de abordaje cualitativo, se realizó con el objetivo de determinar el grado de conocimiento e información que poseen acerca del tema, en aspectos relacionados con el significado, opiniones, sentimientos y emociones, factores de riesgo, identificación y atención a casos. Se contó con la participación de 80 docentes para brindar su percepción social a través de una escala tipo Likert sobre el ASI; asimismo, 20 de ellos/as accedieron a sostener una entrevista semiestructurada para conocer con mayor profundidad su percepción social de este fenómeno. Los resultados reflejaron una conscientización de los y las docentes sobre esta situación, que permitió llegar a la conclusión de que actualmente es un problema muy común que afecta al país. Igualmente, permitió conocer que existe una falta de conocimiento sobre el ASI y la forma de actuar frente a dicha situación, debido a que no son ellos/as quienes llevan de cerca los casos, que en general son atendidos por la mayor autoridad de la escuela.
\end{abstract}

Palabras clave: percepción social - abuso sexual infantil - docentes - escuelas primarias - factores de riesgo.

\section{Abstract}

This qualitative approach research work was conducted with the aim of determining the degree of knowledge and information elementary school teachers in Mérida, Yucatán, Mexico possess on the meaning, opinions, feelings and emotions, risk factors, identification, and attention to cases of child sexual

\footnotetext{
* Estudiante de la licenciatura en Psicología. Facultad de Psicología. Universidad Autónoma de Yucatán. México. mayra.canelaparejas@gmail.com ** Estudiante de la licenciatura en Psicología. Facultad de Psicología. Universidad Autónoma de Yucatán. México.johanagarjona20@gmail.com *** Estudiante de la licenciatura en Psicología. Facultad de Psicología. Universidad Autónoma de Yucatán. México. jazmindzul22@gmail.com **** Estudiante de la licenciatura en Psicología. Facultad de Psicología. Universidad Autónoma de Yucatán. México.michelove-11@hotmail.com ***** Estudiante de la licenciatura en Psicología. Facultad de Psicología. Universidad Autónoma de Yucatán. México. karlasortizc@gmail.com
} 
abuse (CSA). 80 teachers offered their social perception about CSA through a Likert-type scale, and 20 of them agreed to answer a semi-structured interview to learn more about their social perception of this phenomenon. The results reflected an awareness of teachers about this situation, allowing us to conclude that it is a very common problem that currently affects the country, and that there is little knowledge about CSA and how to deal with it, because they are not the ones who provide follow up on these cases, which are usually handled by the school's authorities.

Keywords: social perception - child sexual abuse - teachers - elementary schools - risk factors.

\section{Introducción}

El Abuso Sexual Infantil (ASI) es un tipo específico de maltrato a la infancia que se caracteriza en sus diversas definiciones por dos aspectos fundamentales: la coerción y la asimetría de edad puesto que la o el agresor utiliza la situación de poder que tiene para relacionarse sexualmente con el o la menor y es mayor que su víctima, aunque no necesariamente sea mayor de edad (Gil, 2012).

En un estudio realizado por Acuña (2014), se menciona que el abuso sexual infantil genera consecuencias que pueden observarse en la víctima en un periodo corto de tiempo después de tal acto, estas consecuencias podrían ser de tipo físico, las cuales en la mayoría de las ocasiones son evidentes. También podrían ser psicológicas, estas muchas veces afectan el estado de ánimo, los comportamientos y las relaciones de niños/as, entre otras cosas, y finalmente, se podrían observar problemas de salud que afectan a la víctima.

Así como existen consecuencias de corto plazo en el abuso sexual infantil, también existen consecuencias a largo plazo, entre ellas están los problemas psicológicos, sociales, de salud física, de disfunción sexual y problemas neurobiológicos (Acuña, 2014). Algunas de las consecuencias previamente mencionadas funcionan como indicadores que pueden observar las o los docentes en el niño/a para determinar que algún menor está viviendo una situación de maltrato.

En lo relacionado con los factores de riesgo que podrían propiciar este tipo de maltrato, en una investigación realizada por Redondo, Galdó y García (2008) se menciona que los factores de riesgo del abuso sexual infantil son el conjunto de circunstancias que se dan en entornos sociales, familiares y ambientales en los que se desenvuelve el/la menor, además de las características personales que posea, cuya presencia se relaciona con la probabilidad de que ocurra un caso de abuso sexual infantil.

Los riesgos asociados a los y las niñas: además de que cualquier menor puede ser víctima de abuso sexual infantil, existen algunos factores de riesgo que los vuelven más propensos a sufrir este hecho, tales como la edad y el género; en su gran mayoría son del sexo femenino (entre 2 y 4 veces más frecuente que en hombres). En las niñas suele ocurrir antes de los 12 
años, mientras que en los niños entre los 12 y 13; otro factor es el aspecto físico, ser una hija o hijo no deseado o adoptado, con una alteración o discapacidad física, mental o de desarrollo, tener hiperactividad, mayores necesidades afectivas o ser más expresivo/a. Además, se reconocen algunos factores de riesgo indirectos al estar a cargo de una persona que los cuida en casa, por ejemplo, que esta -ya sea hombre o mujer- sea ajena a la familia, que tenga dificultad para congeniar con el niño/a, incomprensión, poca armonía en el matrimonio, usar métodos disciplinarios fuertes y físicos, aislamiento social de la familia, o que esta persona sea farmacodependiente o alcohólica, tenga un estatus socioeconómico bajo, habite en barrios en condiciones de pobreza, pertenezca a familias numerosas o en desempleo.

Los riesgos asociados al perpetrador: los factores de riesgo personales en el caso de las y los agresores también desempeñan un papel importante a la hora de desarrollar conductas propias de un abusador/a sexual. A lo largo de la historia se han postulado prototipos de abusadores que han caído en la generalización; sin embargo, dicha población es muy diversa y no necesariamente pueden clasificarse como pedófilos. Es posible observar ciertas tendencias y establecer asociaciones respecto a ellos, pero lo cierto es que, así como la víctima puede ser cualquiera, el abusador/a también.

Otros factores que concuerdan con el perfil del abusador/a son, la exposición a la violencia durante la infancia, ya que el hecho de haberse criado y desarrollado en un entorno familiar y social donde se ejercía la violencia puede influir en que en la edad adulta se desarrollen comportamientos que deriven en situaciones de maltrato y abuso sexual, aunado a las distorsiones cognitivas, su poca capacidad o ausencia de empatía o el padecimiento de algún trastorno de personalidad. Cuando los abusos suceden de forma repetitiva, el agresor/a puede sufrir un trastorno de la conducta sexual, como la pedofilia (Redondo et al., 2008).

Este fenómeno es una realidad que existe cotidianamente en la sociedad. Muchos son los niños y las niñas en México que se ven afectados por esta grave problemática, ya sea dentro de su núcleo familiar como fuera de él (Echevarría, como se citó en Giménez, 2017).

Según el Fondo de las Naciones Unidas para la Infancia (UNICEF) (2016b), México está entre los primeros lugares con mayor violencia sexual a nivel mundial. Alrededor de 120 millones de niñas menores de 20 años en todo el mundo ( 1 de cada 10) han experimentado relaciones sexuales por la fuerza $u$ otros actos sexuales forzados, y una de cada tres niñas adolescentes de 15 a 19 años que estuvieron alguna vez casadas (84 millones) ha sido víctima de violencia emocional, física o sexual por parte del esposo o compañero. Asimismo, según la Organización Mundial de la Salud (OMS), uno de cada 13 hombres y una de cada cinco mujeres declaran haber sufrido abuso sexual en la infancia, aunque muy pocos abusos logran comprobarse (UNICEF, 2016a).

En el estado de Yucatán, el abuso sexual infantil es un tema importante debido a que presenta un alto índice en delitos de este tipo y con el paso del tiempo los niveles estadísticos van en aumento. De acuerdo con datos de la Comisión Nacional para Prevenir y Erradicar la Violencia 
Contra las Mujeres de 2018, expuestos por el Observatorio Ciudadano Nacional de la Violencia, Yucatán está por encima de la media nacional ya que, de una muestra de 12,400 niños y niñas, $3.9 \%$ reportó haber sido atacada, pero $2.6 \%$ son yucatecos (Bote, 2019).

En una investigación llevada a cabo por Mendoza (2009) se encontró que, debido a la necesaria función de los/las docentes, debe haber interés por parte de todos frente al abuso sexual infantil, pero no simplemente ante el problema, sino también en la detección y derivación de los diferentes tipos de maltrato en la infancia, los cuales se manifiestan y repercuten en el entorno escolar.

Es importante que el/la docente aprenda a observar a sus alumnos/as tanto en las horas de clases como en su dinámica relacional (los juegos, la salida, etc.) ya que mediante las observaciones registradas, incluidos también otros indicadores como la revelación directa, los comentarios por parte de otros menores o la propia familia, es como puede darse cuenta de que el/ la menor está pasando por una situación de maltrato, y tiene la responsabilidad de intervenir o realizar las derivaciones necesarias (Mendoza, 2009).

Un estudio hecho por Ortega, Baz y Sánchez (2012) menciona que, con independencia de la conducta sexual valorada, la proporción de profesionales que sospecha de un abuso parece ser mayoritaria, pero quienes notifican a las instituciones es minoritaria. La comunidad científica y profesional debe esforzarse por determinar indicadores de abuso sexual que sean funcionales $y$, de la misma forma, no contribuyan a originar sospechas injustificadas.

En la investigación "Percepción de los docentes sobre el Abuso Sexual Infantil en niños y niñas" de Giménez (2017), se obtuvo como resultado que, en relación con el conocimiento que tienen los/las docentes, se puede inferir que hay una definición llena de prejuicios y conceptos ambiguos, identifican claramente que el abuso es hacia el niño o la niña, pero el concepto que tienen no es debido a la búsqueda de información sobre la problemática sino por lo que se escucha cotidianamente sobre el tema. Los/las docentes plantean que en la gran mayoría de los casos el abuso afecta a mujeres, pero saben que igual se puede presentar en varones. Por otra parte, una gran cantidad de docentes mencionan que el abusador/a proviene de la familia de los niños/as o que puede ser alguien de la calle.

Hablar de la evaluación del ASI es una tarea compleja y muy delicada. García (2017), en su estudio menciona que este procedimiento requiere profesionales cualificados, así como de pasos a seguir rigurosos, bien definidos y comprobados empíricamente. Muchos autores/as en sus escritos que hablan sobre la evaluación del ASI dan una importancia considerable a la formación de estos profesionales. Consideran que es importante tener un conocimiento adecuado sobre el ASI, los factores de riesgo y protección, las consecuencias, así como los indicadores que provocan en los/las menores en función de las características del abuso, ya sea de índole intra o extrafamiliar, de su mantenimiento en el tiempo, de las características del abusador/a y de la víctima y del contexto en el que se produce. 
Mendoza (2009), en su estudio "Taller para la detección de casos de Abuso Sexual Infantil en niños de educación básica", encontró que gracias a este taller se identificó que aún existen profesores/as que emplean prácticas de disciplina restrictivas para tratar de establecer hábitos con los/las alumnas, actitudes que dañan la confianza y la relación profesor-alumno respecto a la comunicación, y que impide la construcción de aulas inclusivas.

A partir de lo anterior, se ve la relevancia de los docentes en la vida de los y las niñas. Por ello, este trabajo se abordará con profesionales en educación básica, ya que es de gran importancia realizar un acercamiento con este sector pues, normalmente, son las segundas personas con las que más conviven los/las niñas al pasar varias horas en la escuela y quienes podrían darse cuenta de si está pasando por una situación de ASI, además de brindar cierto apoyo a los/ las menores. La problemática que se quiere estudiar es muy relevante ya que existen muy pocos estudios realizados previamente que nos brinden información acerca de cuál es la percepción que los/las profesoras tienen sobre dicho tema y qué medidas toman o deberían tomar para tratar con niños/as en situaciones de esta magnitud, es decir, que hayan vivido el abuso sexual.

La presente investigación tiene como objetivo conocer cuál es el significado del ASI que poseen los y las docentes en escuelas primarias en Mérida, Yucatán (México); además de descubrir cuáles son sus creencias respecto al ASI, para así lograr identificar los conocimientos que poseen para detectar su presencia en las/los menores. De igual forma, se pretende realizar una descripción de los sentimientos y emociones que se presentan en los docentes cuando hay un caso de ASI y, por último, se espera conocer las medidas que toman ante la presencia de esta situación.

\section{Metodología}

\section{Participantes}

La muestra total del estudiol fueron 80 docentes, a quienes se les aplicó una escala tipo Likert, y 20 de ellos/as accedieron a realizar una entrevista semiestructurada. Estos docentes laboran en escuelas primarias de la ciudad de Mérida, Yucatán, quienes aceptaron participar en la investigación.

El muestreo utilizado para la investigación fue no probabilístico, casos-tipo con la finalidad de contribuir en términos de riqueza y calidad de información (González, 2017), además de que este tipo de muestreo es útil en aquellas investigaciones en las que se pretende analizar los significados, valores, actitudes y creencias de los participantes.

\section{Técnica de recolección de información}

Para la recolección de información se utilizó una entrevista semiestructurada que incluye los siguientes temas: 1) el significado del ASI para los docentes, en el que se abordaron aspectos como el significado, opiniones, emociones y sentimientos; 2) los factores de riesgo, que aborda aspectos personales, sociales y económicos; 3) la identificación del ASI; y 4) la atención de casos, en donde se indagó sobre si poseen preparación para estos casos y si han tenido experiencias. 
Por otro lado, para la parte complementaria se utilizó una escala tipo Likert, tomada de un estudio realizado por Priegue y Cambeiro (2016), "Los conocimientos acerca del maltrato infantil de los futuros profesionales de la educación: un estudio exploratorio". Por lo tanto, al ser una escala que mide el maltrato infantil, fue adaptada de acuerdo con los objetivos de esta investigación que pretende medir la percepción social sobre el abuso sexual infantil. Asimismo, se eliminaron los ítems que no pertenecían a los objetivos del presente trabajo. En total, el instrumento se componía de 82 ítems integrados en 8 dimensiones: datos de identificación, formación sobre maltrato infantil, conocimiento sobre el maltrato infantil, contacto con casos de maltrato infantil en la familia, factores de riesgo del maltrato infantil en la familia, indicadores de maltrato infantil en la familia, creencias e interés académico hacia el maltrato infantil. De esta forma, se cambiaron algunas palabras por sinónimos para adaptarlo al entorno social de Mérida, ya que esta prueba se desarrolló en España. Después de realizar las modificaciones antes mencionadas, la encuesta quedó conformada por un total de 47 ítems y 3 dimensiones: la primera dimensión, referente a los factores de riesgo del ASI, tiene 9 ítems; la segunda fue sobre indicadores del ASI, contó con 18 ítems; y la última dimensión contiene 20 ítems acerca de las creencias y actitudes en torno al tema.

\section{Procedimiento}

El procedimiento para llevar a cabo la investigación fue el siguiente: lo primero fue la introducción a escuelas primarias en Mérida, en las cuales se realizó la investigación. Se les invitó a participar de manera voluntaria en la aplicación del instrumento y la recopilación de información mediante la técnica de entrevista semiestructurada sobre abuso sexual infantil. Las estrategias de acceso al campo se dieron mediante permisos brindados por las y los directores de las escuelas y por las/los docentes.

Se procedió a proporcionar el consentimiento informado, para que las/los docentes tomaran la decisión sobre su participación. En dicho consentimiento se plantearon los lineamientos a seguir durante la realización y aplicación de instrumentos y técnicas, para que la introducción al escenario de estudio, el proceso de recolección de información y la colaboración de los/las participantes fuera voluntario y adecuado, sin ningún tipo de problema; una importante aclaración en el documento fue que toda la información sería confidencial y anónima, para que los participantes conocieran las consideraciones éticas sobre el uso de los datos que se obtendrían en la investigación.

Posteriormente, en un primer momento se realizó la entrevista sobre ASI, que contenía cuatro temas. Enseguida, se procedió a la aplicación de la encuesta. Después de recopilar la información se capturaron los datos obtenidos y, finalmente, se realizó el análisis de información y el reporte de los resultados de la investigación. 


\section{Resultados}

Los resultados del estudio se obtuvieron a través de los datos de las 20 entrevistas semiestructuradas y de los cuestionarios respondidos por 80 docentes de escuelas primarias de la ciudad de Mérida, que conformaron la muestra.

En las entrevistas se abordaron cinco dimensiones: el significado (significado, opinión y sentimientos y emociones), factores de riesgo (familiares, sociales y socioeconómicos), identificación (los indicadores del ASI), formas de actuación (qué hacen cuando hay un caso de ASI) y, por último, experiencias (si han detectado casos de ASI o no).

Con respecto a la escala tipo Likert, todos los resultados obtenidos se analizaron en el software de análisis estadístico SPSS versión 25.0. De los/las participantes, 65 eran mujeres (81\%) y 15 hombres (18.8\%). De acuerdo con el tipo de escuela, 63 pertenecen al sistema público (78.8\%) y 17 provienen de escuelas privadas (21.3\%). Por último, con respecto al turno, 47 docentes son del turno matutino (58.8\%) y 33 del vespertino (41.3\%). La recolección de datos se llevó a cabo durante los meses de octubre-septiembre de 2019.

En cuanto a la entrevista semiestructurada, en la dimensión del significado del abuso sexual infantil que tienen los/las docentes entrevistados se encontró lo siguiente:

De acuerdo con sus respuestas, el abuso sexual infantil significa tocar o ultrajar el cuerpo y las partes íntimas de niños o niñas, con o sin penetración, obligándoles a realizar acciones sin su consentimiento. También mencionan que es un tipo de violencia o maltrato que por lo general es físico y lo lleva a cabo una persona adulta hacia niñas, niños o adolescentes menores de 18 años (véase la tabla 1).

En lo que se refiere a las opiniones, se menciona que es un tema fuerte y que es algo malo que no debería existir ya que este fenómeno provoca consecuencias en las víctimas, lo cual se ve reflejado en diferentes ámbitos de su vida, como en los familiares, escolares, sociales, e incluso puede tener efectos cuando llegan a la etapa adulta. Las y los docentes se encuentran en desacuerdo con este tema y se plantea que hay ignorancia de las personas por tales situaciones y que la sociedad aún sigue siendo un poco cerrada ante problemas como el Abuso Sexual Infantil (véase la tabla 1).

Con respecto a las emociones que les provoca el ASI, las y los docentes refieren predominantemente la tristeza por la ocurrencia de estas situaciones, al igual que por los niños y niñas que pasan por algún caso de abuso sexual; otras de las emociones que registran fueron: enojo, miedo y asco. Por otro lado, en cuanto a los sentimientos se encontró el coraje, dolor, impotencia, pena, furia, preocupación, repugnancia, horror, sufrimiento y compasión, siendo la más predominante el coraje, lo cual se presenta al ver que ocurran estas situaciones; la impotencia y preocupación se menciona que lo sienten al enterarse de casos y no poder intervenir de manera profunda ante ello, y los sentimientos como dolor, pena, sufrimiento y compasión se presenta hacia los y las niñas que pasan por tales situaciones, por el contrario, la furia y repugnancia lo sienten hacia los agresores (tabla 1). 
Tabla 1. Respuestas de los y las docentes que abarcan la dimensión de significado sobre el ASI Significado del Abuso Sexual Infantil

"¿Qué significa para mí? El abuso sexual es violentar en la persona física [...], pues si estamos hablando de abuso infantil, pues estamos hablando entonces de los niños ¿no?, es como ultrajar o violar, manejar algo que el niño no quiere, el niño o niña no quiere sobre su cuerpo."

"Pues es un tipo de violencia hacia un menor, una manera de pasar sobre su integridad física, emocional y psicológica que, pues le hace mucho daño, o sea a su desarrollo personal, eso es lo que sería para mí".

"El abuso sexual infantil sería provocar en el niño algún tipo de situación en el que pueda sentirse incómodo con respecto a su cuerpo".

"Pues es cuando a un pequeño se le priva de su sexualidad, en cuestión de que, pues hay un tocamiento, un acercamiento sexual, no tiene que ser siempre penetración o violación, sino que solo contacto con sus partes íntimas lo priva de su libertad sexual".

"Pues el abuso sexual infantil es una acción que ejerce un individuo hacia otra persona sin consentimiento alguno, que puede ser el frotamiento o penetración hacia una parte del cuerpo".

"El abuso infantil, ok, el abuso sexual es todo lo que va en contra de la integridad de algún niño, niña sí, no solamente porque es niña quiere decir que se van a fijar principalmente los agresores en esa niña si no que incluye desde niña hasta el niño... el abuso sexual es la falta de conciencia que tiene las personas y que se integran vamos a decir em, vamos a decir que corrompen la privacidad, más que nada la intimidad de algunos niños, que puede ser tanto físico como emocionalmente".

\section{Opinión que tienen del Abuso Sexual Infantil}

"Es un tema muy fuerte en el cual estoy muy en desacuerdo, porque es la infancia o la niñez, es una etapa muy bonita en la cual hay que respetar, valorar cuidar, fomentar y pues es algo que, la gente creo que no tiene cerebro, sentimientos al hacerlo y pues yo, estoy en total desacuerdo con esas personas que, creo que no tuvieron la infancia adecuada para poder, para hacer las cosas negativas que hacen, sobre todo con los niños".

"Pues que es malo... pues no estoy de acuerdo y estoy desacuerdo con esas personas".

"Que es algo horrible, es algo feo, algo que no debe de existir... o sea estamos muy arraigados, somos muy ignorantes en ese tema, entonces sí da mucho trabajo y todavía la gente le da trabajo hablar con las personas sobre este tema, entonces no tenemos la educación para resolver eso".

"Pues que es algo grave, porque realmente a un niño lo privas de su naturaleza, que es su infancia, su inocencia, entonces el niño a veces crece con problemas psicológicos, problemas de integración, de comunicación, y realmente el niño se siente como que retraído y puede provocarle algo más a él en cuanto a su persona".

"Opino que de verdad es negativo, es malo porque esté, el menor es el que termina recibiendo todo lo que es este, los problemas ¿no?, empiezan a tener problemas ya sea en la casa, en la escuela o en la calle".

"Pienso que es malo... entonces es malo y se sale de control porque muchas veces, como les había mencionado, es algo grave que puede afectar a los niños, por ejemplo, si un niño es abusado sexualmente no quiere decir que en una semana ya se le olvidó algo, si no que puede traer secuelas, entonces el niño puede quedar traumado y eso le puede afectar no solo en su infancia sino en su adultez".

\section{Emociones y sentimientos acerca del Abuso Sexual Infantil}

"A mí, me da coraje, mucho coraje, dolor, me dan ganas de golpear a la persona que escucho que hace eso, me da enojo, impotencia porque pues no puedes hacer nada...".

"Horror, asco, coraje e impotencia... da mucha pena ver cómo está la situación tan aberrante en los niños que son maltratados sexualmente".

"Pues me daría tristeza por la persona, no sé, repugnancia hacia el violador".

"Pues a mí me da tristeza, a mí me da mucha tristeza, porque un niño para mí son angelitos...".

"El tema me causa enojo y molestia cuando... a mí me enoja y me molesta mucho que sucedan esas cosas".

"A mí me provoca enojo por lo mismo que no se hace nada, enojo y tristeza, tristeza en pensar y ponerme en el lugar de los niñitos que sufren de estos abusos, enojo por parte de la autoridad que no hacen nada para detener estos casos". 
Según las y los docentes, en la dimensión de factores que propician el ASI consideran que son los siguientes: la mayoría de las/los docentes respondieron que dentro de los factores familiares que propician el abuso sexual infantil se encuentra, en primera instancia, que el o la abusadora haya tenido una infancia negativa y una mala educación, al igual que el descuido y la falta de comunicación entre padres y madres con sus hijos e hijas ya que ellos muchas veces llegan tarde de trabajar y los dejan solos durante el día o con algún familiar (tíos/as, primas/os, hermanos/as), con personas desconocidas o vecinos/as. Por último, otro factor que afecta el ámbito familiar son la dispersión familiar y las segundas nupcias, porque muchas veces son los padrastros o madrastras quienes abusan sexualmente de el menor ya que los padres/madres confían más en sus parejas y los ponen por encima de sus hijos/hijas (tabla 2).

Respecto a las respuestas que se encontraron de los factores sociales en los/las docentes, dicen que el principal factor que propicia el ASI es el consumo de drogas y sustancias de cualquier tipo; otro factor importante es el bullying, ya que muchas veces los mismos compañeros o compañeras abusan físicamente en ese tipo de situaciones, donde puede haber tocamiento o incluso una penetración en sus partes íntimas. De igual manera, la ignorancia de las personas ante el abuso es otro de los factores sociales, debido a que muchas veces no se toman en cuenta como una situación grave o la dejan pasar como si fuese algo normal, cuando no es así. Por último, otro factor que la mayoría considera es la falta de consciencia por parte de la sociedad, o incluso en la misma familia, porque la educación comienza desde casa y es ahí donde se tiene que aprender a respetar la integridad de las personas (tabla 2).

Con base a las respuestas encontradas en los factores socioeconómicos, la mayoría de las y los docentes piensa que puede ser un factor y a la vez no, puede ser porque muchas veces ambos padres tienen que salir a trabajar por necesidad o por problemas económicos. Algunos/ as docentes piensan que el nivel socioeconómico influye, porque es en las personas de bajos recursos que mayormente se da el abuso sexual debido a que quienes tienen poder influyen sobre las personas de menor poder, comprándolas con dinero para satisfacer sus necesidades (tabla 2). 
Tabla 2. Respuestas de los y las docentes que abarcan la dimensión de factores de riesgo

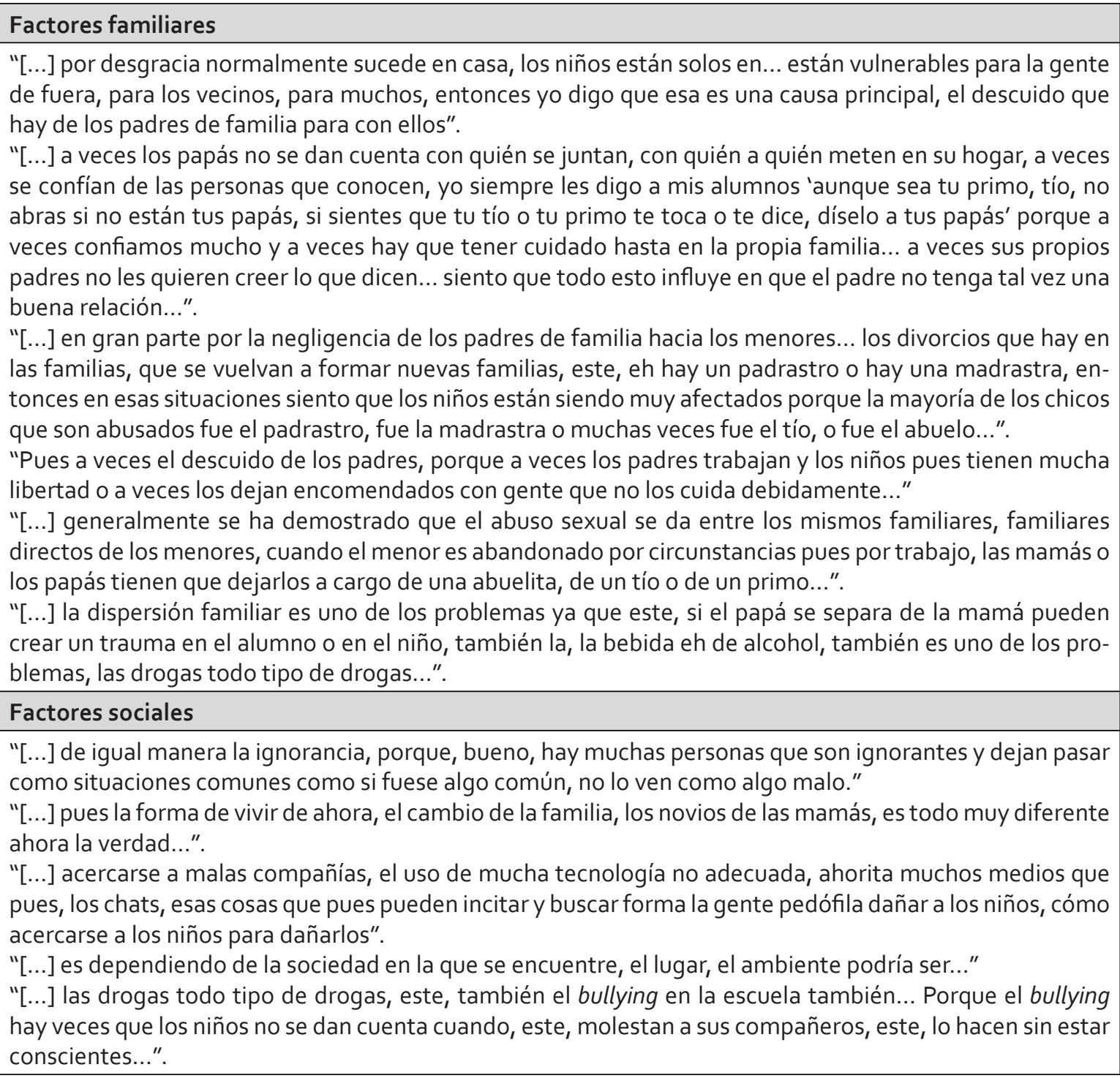




\begin{tabular}{l}
\hline Factores socioeconómicos \\
"[...] desafortunadamente nos toca más a la gente que tiene recursos económicos muy bajos porque no sa- \\
ben defenderse, no pueden defenderse y el terror a que les hagan más daño, prefieren callarlo y ocultarlo". \\
"El factor económico, no, no depende, yo creo que depende más de una falta de educación... el factor de \\
que una familia tenga problemas económicos causa muchos más problemas que un abuso sexual, tiene que \\
trabajar la mamá, dejar solo al niño, es una cosa que indirectamente sí afecta, pero no es la razón en si". \\
"El universo económico no sé si puede influir también y no, porque hay personas que pueden tener dinero, \\
pero tienen un problema psicológico o de alcohol o droga que pueda afectar, a lo mejor se pudiera dar más \\
en situaciones de bajos recursos, pues probablemente por la falta de educación..." \\
"Podría ser, porque muchas veces las personas con escasos recursos pues tienen que salir a trabajar ¿no? \\
Pues el factor de ¿con quién dejo a? y ¿cómo le hago con?, trabajo todo el día, entonces dejamos a los hijos \\
encomendados con la prima, vecina o hasta solos en casa, para salir a trabajar, para buscar qué hacer; en- \\
tonces sí hay ciertas limitantes..." \\
"Yo creo que no al cien, o sea porque realmente pasan estos problemas con gente que tiene dinero, co- \\
loquialmente, pero a veces creo que un 50 sí, porque son niños que sus papás trabajan y pues no están \\
cuidándolos..." \\
"En lo económico a veces se abusa de los niños a través del chantaje con el dinero o a través de objetos de \\
valor, 'te voy a dar un celular, pero dejas que yo te haga esto', el poner dinero también propicia que la gente \\
que tenga poder abusa de la gente que no..." \\
"No tanto es el factor económico sino es dependiendo de la sociedad en la que se encuentre, el lugar, el \\
ambiente podría ser, no porque sí es, vamos a llamarlo pobre, va a sufrir, no, porque incluso sucede en los \\
que son, los que tienen mayor estabilidad económica..."
\end{tabular}

Para la dimensión de identificación de casos de ASI se encontró lo siguiente: con respecto a cómo los y las docentes identifican el abuso sexual infantil, la mayoría de coincidió en que la conducta del niño/a es uno de los detonantes más importantes, y que por lo general se muestran retraídos, tienen miedo y más que nada, hay un cambio en la conducta ya que conocen a sus alumnos/as, y observan de un momento a otro que tienen una conducta retraída, no quieren relacionarse con sus compañeros, y en ocasiones tienen una conducta alterada; igualmente, mencionan que muchas veces dejan de asistir o van con la misma ropa, se ven decaídos o incluso se duermen o se dan cuenta de que dejan de comer; otro punto importante es que ya no realizan sus tareas. Por otro lado, hay docentes que se dan cuenta porque los y las niñas se acercan a los docentes y se lo dicen, pero en otras, lo comentan por los temas que están viendo en clase. Además, los docentes se dan cuenta de que algo está pasando por marcas físicas visibles que tienen, por lo tanto, realizan investigaciones para saber qué está pasando y muchas veces tienen que apoyarse de la Unidad de Servicio de Apoyo a la Educación Regular (USAER) (tabla 3). 
Tabla 3. Respuestas de los y las docentes que abarcan la dimensión de indicadores del ASI

\begin{tabular}{|c|}
\hline \\
\hline 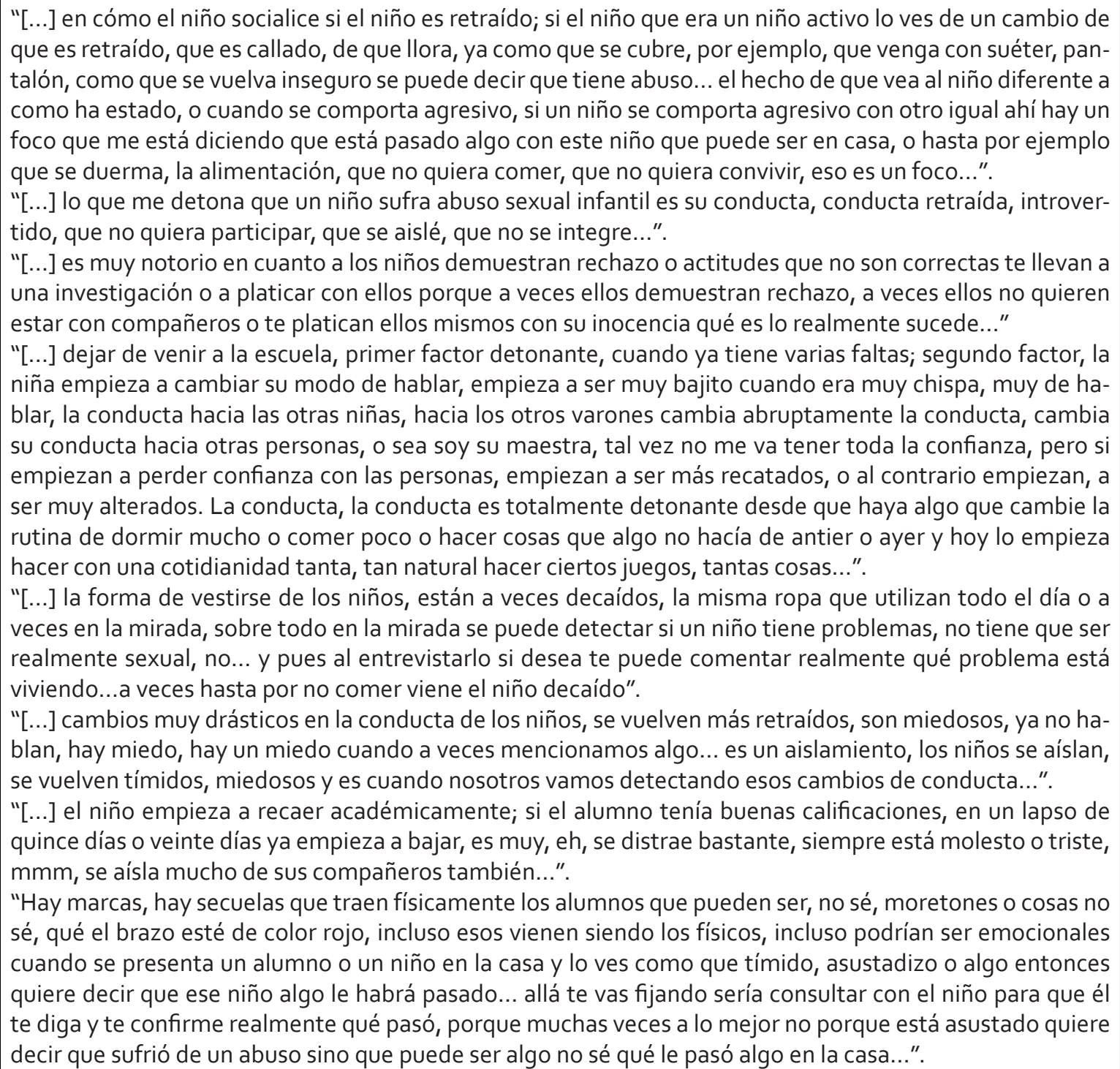 \\
\hline
\end{tabular}

En la dimensión de formas de actuar que llevan a cabo los y las docentes al identificar un caso de ASI reportan las siguientes: de acuerdo con las respuestas, se puede observar que la mayoría concuerda acerca de lo que se tiene que hacer cuando se presenta un caso de abuso sexual infantil, mencionando en primera instancia que deben seguir el protocolo de actuación que poseen, el cual estipula que lo primero que hay que hacer es comunicarle todo al director/a para que sea quien se haga cargo de la situación, en conjunto con el/la psicóloga de la escuela la USAER o, en casos extremos, con las autoridades judiciales pertinentes (tabla 4). 
Tabla 4. Respuestas de los y las docentes que abarcan la dimensión de formas de actuar ante un caso de ASI

\section{Formas de actuar}

"En la Secretaría se nos tiene otorgado un protocolo de seguimiento para cuando se presentan este tipo de situaciones... se tiene y se debe seguir el protocolo en el cual tú te enteras, redactas lo que te enteraste, informas a tu superior, tu superior tiene que, por ejemplo, hacer el llamado a los padres de familia, contigo maestro, con el niño y no se pregunta dos veces al menor la situación, se pregunta una vez cuando tú te enteras de la situación, es todo...".

"[...] lo identificamos, se lo pasamos a la directora y ella es la que sigue el protocolo para esto, la verdad, se le habla a otras instancias para que ellos vengan o se manda allá a la casa para que ellos investiguen realmente la situación del niño".

"[...] se le informa al director, se le tiene que hacer un documento donde se le escriba todo, tal y como está toda la situación que se presenta del niño, así como él lo vaya contando se va anotando la información para tener un expediente o una prueba y ya pues trabajar con las autoridades pertinente para que se pueda abrir esta situación".

"Nosotros aquí en la escuela tenemos un protocolo de actuación, entonces la USAER tiene el papel de apoyar a la escuela porque el director es el que tiene la responsabilidad en este caso de levantar la denuncia, entonces la USAER tiene la obligación de apoyar y guiar qué es lo que se va a hacer, no nos metemos adentro ni averiguamos, solo con lo que sabemos porque protegemos al menor, entonces nosotros lo llevamos a las instancias que deben de ser y ahí ellos actúan, ya nosotros no, o sea solo guiamos y apoyamos".

"[...] realmente tú no eres policía, tú no vas a investigar si es cierto o no, pero hay un protocolo de actuación que los docentes llenamos, que llenamos con la situación de lo que sucede en el momento que la niña te cuente su versión y luego eso se lleva al jurídico de la SEP, al jurídico penal, a la Secretaría de Educación también... nosotros solo actuamos con el protocolo de actuación y cumplimos y realmente la gente especializada, los psicólogos, la gente del jurídico son los que investigan si realmente sucedió o no, pero no podemos quedarnos callados...".

"[...] nosotros lo único que hacemos es tratar de canalizarlo a autoridades que correspondan, aquí lo primero, si sucede en la escuela es a través del servicio de USAER, si lo detectamos y lo comprobamos... y es mandar a buscar a la mamá para que la mamá o el papá para que se enteren primero que tienen el apoyo de la escuela..., pero ya el aspecto legal ya corresponde a este otro tipo de autoridades, ya la escuela no puede hacer nada, ya caen en un ámbito judicial".

"Pues nosotros, como el protocolo que tiene la escuela, o sea que nos han dado en nuestros cursos de sala con nuestro director o el que esté a cargo de la escuela y notificarle cómo es la situación del alumno... el director es el que manda a hablar a los papás o en su caso, habla a las dependencias correspondientes que vean esto".

"[...] con base a lo que he escuchado, he visto y me he informado un poquito en noticias, internet y todo lo demás, lo que se hace en estos casos es acudir a las autoridades, primeramente, con la madre del menor, con el tutor y en el caso que no tenga mamá, papá... es acudir con el tutor y posteriormente platicar con ella, con un psicólogo incluso con las autoridades...".

Por último, en la dimensión de experiencias se observó lo siguiente: algunos docentes mencionan que sí han tenido mínimo una experiencia, otros las han escuchado, y unos cuantos mencionaron que no han pasado por este tipo de situaciones, ya sea porque no se les ha presentado el caso o debido a que tienen poca experiencia laboral (tabla 5).

Diólo os sobre Educación temas ACtUales EN INVESTIGACION EDUCATIVA 
Tabla 5. Respuestas de los y las docentes que abarcan la dimensión de experiencias sobre el ASI

\begin{tabular}{l}
\hline Experiencias \\
"[...] en la escuela no he tenido situación de mis casi 14 años de servicio, no he tenido una situación de abu- \\
so, pero sí he escuchado, pues en lo que lees o lo que te cuentan...". \\
"Yo sí tuve una experiencia así con un alumno, hace como 15 años. \\
[...] me ha tocado en otras escuelas donde el abuso infantil ha sido fuerte que hemos descubierto niños en \\
obras de teatro... que han sido violadas... \\
"De mi grupo como tal, miren hace como dos años, yo estaba dando sexto grado precisamente y al final en \\
el último mes de clase, yo tuve a una chica que dejó de venir y de repente viene la, viene la mamá y me dice: \\
"Maestra va a estar faltando unos días", porque sucedió algo que no quisiera ella que hubiera sucedido y me \\
contó de manera personal que la chica había estado siendo abusada por su padrastro...". \\
"[...] me pasó un caso con una alumna hace dos años y sí me metí un poquito en el tema...". \\
"Yo fui el año pasado director de la escuela y hubo un caso parecido, pues la niña comentó a una maestra \\
que vivió un abuso sexual, pero por tocamiento, porque está también clasificado, entonces uno tiene que \\
intervenir primero por la integridad del niño....". \\
"No, nosotros no tenemos experiencia, la experiencia que nos da, por ejemplo, yo tengo 42 años de servicio \\
entonces he visto, he visto algunas situaciones, pero como escuela no podemos intervenir directamente...". \\
"Eh, no, la verdad no, o sea sí he visto embarazos de alumnos que se dan a una temprana edad, pero no, no \\
recaen en abuso sexual". \\
"Hasta ahorita no he tenido ninguna experiencia...".
\end{tabular}

Con respecto a la escala tipo Likert, se obtuvieron los siguientes resultados en cada reactivo. Los resultados se encuentran divididos en tres dimensiones: la primera es de factores de riesgo, seguido de indicadores $y$, por último, creencias y actitudes.

Uno de los aspectos de interés son los factores de riesgo que los y las docentes consideran que propician el abuso sexual infantil. Para ello, tenían que señalar en cada reactivo si estaban de acuerdo (A), totalmente de acuerdo (TA), en desacuerdo (D), y totalmente en desacuerdo (TD), respecto a la influencia de dichos factores en cuanto a la ocurrencia de casos de ASI. En la tabla 6 se puede observar que los factores de riesgo con los que más estuvieron de acuerdo los y las docentes fueron los de índole familiar (estilos parentales, desestructuración familiar, malos tratos en la infancia de los padres, conflictos, violencia en la pareja, aunque la presencia de factores de riesgo no implica el abuso sexual en la familia); por debajo se encuentran los factores sociales (la aprobación social de la violencia como forma de resolver los problemas, falta de redes de apoyo social y el estrés); y por último, están los factores conductuales y de salud (con enfermedades, minusvalías o problemas de conducta, problemas de sueño y alimentación).

En cuanto a los indicadores que los y las docentes consideran como señales de abuso sexual infantil, se encontró que le dan más importancia a los de índole conductual (ausentismo escolar, trabajos impropios a su edad, actitudes defensivas, conocimientos y conductas sexuales, cansancio y tendencia a dormirse en clase, y trato desigual entre hermanos y hermanas); enseguida se encuentran los familiares (falta de supervisión parental, relación fría entre padre, 
madre y menor, rechazo al hablar de problemas de el o la menor, escasa relación familia-escuela); seguido por lo cognitivo (desarrollo madurativo inferior, dificultades de aprendizaje y problemas de desarrollo cognitivo); continuando con los indicadores sociales (falta de empatía hacia los demás y relaciones distantes con los educadores/as; y finalizando con indicadores físicos (heridas, moretones, quemaduras y misma ropa o ropa inadecuada) (véase la tabla 6).

En cuanto a las creencias y actitudes, los y las docentes mostraron mayor contundencia en los reactivos que tratan sobre "el abuso sexual infantil en la familia es un delito", de la cual $80 \%$ estuvo "totalmente de acuerdo"; el siguiente reactivo con el que la mayoría estuvo totalmente de acuerdo fue "en el entorno educativo debe existir un protocolo de denuncia y actuación del abuso sexual infantil en la familia" con 73.8\%; y finalmente, el último reactivo más significativo es "el abuso sexual infantil en la familia no existe en las clases sociales altas"; en tal reactivo 71.3\% estuvo "totalmente en desacuerdo". Por otra parte, en los reactivos que se encontró mayor dispersión en las respuestas son "el abuso sexual infantil es fácil de reconocer", "las consecuencias del abuso sexual infantil son diferentes según el sexo", "el ámbito escolar es un lugar privilegiado para detectar situaciones de riesgo de abuso sexual infantil en la familia" y "la mayoría de los agresores responsabiliza del abuso sexual al menor" (tabla 6).

Tabla 6. Resultados de los y las docentes en la escala tipo Likert sobre los factores de riego; indicadores; y creencias y actitudes del Abuso Sexual Infantil

\begin{tabular}{|l|l|l|l|l|}
\hline Factores de riesgo del Abuso Sexual Infantil en la familia & TD & D & A & TA \\
\hline $\begin{array}{l}\text { Los menores con enfermedades, minusvalías o problemas de conduc- } \\
\text { ta tienen mayor riesgo de sufrir abuso sexual. }\end{array}$ & $7.5 \%$ & $23.8 \%$ & $\mathbf{4 3 . 8 \%}$ & $\mathbf{2 5 \%}$ \\
\hline $\begin{array}{l}\text { Los estilos parentales negligente y autoritario correlacionan positiva- } \\
\text { mente con el abuso sexual infantil. }\end{array}$ & $3.8 \%$ & $\mathbf{2 6 . 3 \%}$ & $\mathbf{5 0 \%}$ & $\mathbf{2 0 \%}$ \\
\hline $\begin{array}{l}\text { La aprobación social de la violencia como forma de resolver los pro- } \\
\text { blemas de relación constituye un factor sociocultural de riesgo del } \\
\text { abuso sexual infantil. }\end{array}$ & $3.8 \%$ & $\mathbf{1 1 . 3} \%$ & $\mathbf{5 5 \%}$ & $\mathbf{3 0 \%}$ \\
\hline $\begin{array}{l}\text { La desestructuración familiar se considera un factor de riesgo para el } \\
\text { abuso sexual infantil. }\end{array}$ & $\mathbf{1 0 \%}$ & $\mathbf{2 1 . 3 \%}$ & $\mathbf{4 7 . 5 \%}$ & $\mathbf{2 1 . 3 \%}$ \\
\hline $\begin{array}{l}\text { La falta de redes de apoyo social y el estrés son factores de riesgo } \\
\text { importantes en las situaciones de abuso sexual infantil. }\end{array}$ & $3.8 \%$ & $\mathbf{2 8 . 7 \%}$ & $\mathbf{4 8 . 8 \%}$ & $\mathbf{1 8 . 8 \%}$ \\
\hline $\begin{array}{l}\text { Una historia de malos tratos en la infancia de los padres constituye un } \\
\text { factor de riesgo para convertirse en abusador en la edad adulta. }\end{array}$ & $6.3 \%$ & $\mathbf{2 2 . 5 \%}$ & $\mathbf{4 5 \%}$ & $\mathbf{2 6 . 3 \%}$ \\
\hline $\begin{array}{l}\text { Los conflictos y la violencia en la pareja pueden derivarse en episodios } \\
\text { de abuso sexual hacia los hijos. }\end{array}$ & $5 \%$ & $30 \%$ & $\mathbf{5 2 . 5 \%}$ & $\mathbf{1 2 . 5 \%}$ \\
\hline $\begin{array}{l}\text { Un factor individual de riesgo de abuso sexual en los menores es la } \\
\text { presencia de problemas de sueño y alimentación. }\end{array}$ & $8.8 \%$ & $\mathbf{3 7 . 5 \%}$ & $\mathbf{3 3 . 8 \%}$ & $\mathbf{2 0 \%}$ \\
\hline $\begin{array}{l}\text { La presencia de factores de riesgo no significa automáticamente la } \\
\text { aparición de abuso sexual infantil en la familia. }\end{array}$ & $3.8 \%$ & $\mathbf{1 7 . 5} \%$ & $\mathbf{5 2 . 5} \%$ & $\mathbf{2 6 . 3 \%}$ \\
\hline
\end{tabular}




\begin{tabular}{|c|c|c|c|c|}
\hline Indicadores del Abuso Sexual Infantil & TD & D & A & TA \\
\hline Heridas, moretones, quemaduras... & $3.8 \%$ & $21.3 \%$ & $47.5 \%$ & $27.5 \%$ \\
\hline Misma ropa o ropa inadecuada a la estación del año. & $7.5 \%$ & $42.5 \%$ & $43.8 \%$ & $6.3 \%$ \\
\hline Absentismo escolar raramente justificado. & $6.3 \%$ & $27.5 \%$ & $48.8 \%$ & $17.5 \%$ \\
\hline Realización de trabajos impropios a su edad. & $3.8 \%$ & $23.8 \%$ & $55 \%$ & $17.5 \%$ \\
\hline Actitudes defensivas ante cualquier aproximación física. & $1.3 \%$ & $3.8 \%$ & $52.5 \%$ & $42.5 \%$ \\
\hline Falta de supervisión parental. & $1.3 \%$ & $28.7 \%$ & $48.8 \%$ & $21.3 \%$ \\
\hline Conocimientos y/o conductas sexuales no adecuados a su edad. & $1.3 \%$ & $7.5 \%$ & $51.2 \%$ & $40 \%$ \\
\hline $\begin{array}{l}\text { Problemas médicos y ausencias prolongadas o repetidas en el tiem- } \\
\text { po. }\end{array}$ & $3.8 \%$ & $27.5 \%$ & $55 \%$ & $13.8 \%$ \\
\hline Cansancio / tendencia a dormirse en clase. & $6.3 \%$ & $42.5 \%$ & $38.8 \%$ & $12.5 \%$ \\
\hline Despedidas / reencuentros fríos entre padres y menor. & $7.5 \%$ & $37.5 \%$ & $46.3 \%$ & $8.8 \%$ \\
\hline Trato desigual hacia los hermanos. & $2.5 \%$ & $45 \%$ & $38.8 \%$ & $13.8 \%$ \\
\hline Falta de empatía hacia lo que sienten los demás. & $3.8 \%$ & $56.3 \%$ & $32.5 \%$ & $7.5 \%$ \\
\hline Rechazo a hablar de los problemas del menor. & $2.5 \%$ & $15 \%$ & $66.3 \%$ & $16.3 \%$ \\
\hline Escasa relación familia-escuela. & $3.8 \%$ & $32.5 \%$ & $51.2 \%$ & $12.5 \%$ \\
\hline $\begin{array}{l}\text { Desarrollo madurativo inferior al correspondiente a su estadio evo- } \\
\text { lutivo. }\end{array}$ & $3.8 \%$ & $42.5 \%$ & $41.3 \%$ & $12.5 \%$ \\
\hline Relaciones distantes con los educadores. & $2.5 \%$ & $32.5 \%$ & $51.2 \%$ & $13.8 \%$ \\
\hline Dificultades de aprendizaje. & $3.8 \%$ & $33.8 \%$ & $48.8 \%$ & $13.8 \%$ \\
\hline Problemas en el desarrollo cognitivo. & $8.8 \%$ & $41.3 \%$ & $35 \%$ & $15 \%$ \\
\hline Creencias y actitudes en torno al Abuso Sexual Infantil & TD & D & A & TA \\
\hline $\begin{array}{l}\text { El abuso sexual sólo afecta a niñas/chicas con comportamientos ina- } \\
\text { daptados. }\end{array}$ & $56.3 \%$ & $38.8 \%$ & $1.3 \%$ & $3.8 \%$ \\
\hline Los menores a menudo provocan y seducen a los adultos. & $68.8 \%$ & $26.3 \%$ & $3.8 \%$ & $1.3 \%$ \\
\hline $\begin{array}{l}\text { El abuso sexual infantil en la familia no existe en las clases sociales } \\
\text { altas. }\end{array}$ & $71.3 \%$ & $25 \%$ & $1.3 \%$ & $2.5 \%$ \\
\hline El abuso sexual infantil en la familia es un asunto privado. & $62.5 \%$ & $25 \%$ & $7.5 \%$ & $5 \%$ \\
\hline $\begin{array}{l}\text { El profesorado no debe intervenir ante la sospecha de abuso sexual } \\
\text { infantil. }\end{array}$ & $63.7 \%$ & $31.3 \%$ & $3.8 \%$ & $1.3 \%$ \\
\hline El abuso sexual infantil en la familia es un delito. & $8.8 \%$ & $0 \%$ & $11.3 \%$ & $80 \%$ \\
\hline $\begin{array}{l}\text { En los centros educativos debe existir un protocolo de denuncia y ac- } \\
\text { tuación del abuso sexual infantil en la familia. }\end{array}$ & $8.8 \%$ & $2.5 \%$ & $15 \%$ & $73.8 \%$ \\
\hline Los padres tienen poder absoluto sobre sus hijos. & $35 \%$ & $43.8 \%$ & $15 \%$ & $6.3 \%$ \\
\hline $\begin{array}{l}\text { Cualquier persona (profesional o no) debe notificar una sospecha de } \\
\text { abuso sexual infantil. }\end{array}$ & $7 \cdot 5 \%$ & $1.3 \%$ & $27.5 \%$ & $63.7 \%$ \\
\hline $\begin{array}{l}\text { Los padres que abusan sexualmente a sus hijos tienen problemas } \\
\text { mentales, de alcohol o de drogas. }\end{array}$ & $10 \%$ & $18.8 \%$ & $36.3 \%$ & $35 \%$ \\
\hline $\begin{array}{l}\text { El papel del profesorado es fundamental en la detección y notifica- } \\
\text { ción del abuso sexual infantil. }\end{array}$ & $1.3 \%$ & $8.8 \%$ & $43.8 \%$ & $46.3 \%$ \\
\hline
\end{tabular}




\begin{tabular}{|c|c|c|c|c|}
\hline El abuso sexual es fácil de reconocer. & $7.5 \%$ & $55 \%$ & $33.8 \%$ & $3.8 \%$ \\
\hline El abuso sexual infantil en la familia es un fenómeno aislado. & $33.8 \%$ & $57.5 \%$ & $7.5 \%$ & $1.3 \%$ \\
\hline $\begin{array}{l}\text { El ámbito escolar es un lugar privilegiado para detectar situaciones de } \\
\text { riesgo de abuso sexual infantil en la familia. }\end{array}$ & $6.3 \%$ & $23.8 \%$ & $55 \%$ & $15 \%$ \\
\hline $\begin{array}{l}\text { La mayoría de los casos de abuso sexual infantil se producen fuera del } \\
\text { ámbito familiar. }\end{array}$ & $25 \%$ & $61.3 \%$ & $10 \%$ & $3.8 \%$ \\
\hline La mayoría de los agresores responsabiliza del abuso sexual al menor. & $16.3 \%$ & $28.7 \%$ & $35 \%$ & $20 \%$ \\
\hline $\begin{array}{l}\text { Actualmente existe una mayor sensibilización social frente al abuso } \\
\text { sexual infantil en la familia. }\end{array}$ & $5 \%$ & $22.5 \%$ & $56.3 \%$ & $16.3 \%$ \\
\hline $\begin{array}{l}\text { La naturaleza humana impulsa a los padres a cuidar y querer a sus } \\
\text { hijos. }\end{array}$ & $2.5 \%$ & $6.3 \%$ & $48.8 \%$ & $42.5 \%$ \\
\hline $\begin{array}{l}\text { Las consecuencias del abuso sexual infantil son diferentes según el } \\
\text { sexo. }\end{array}$ & $30 \%$ & $33.8 \%$ & $32.5 \%$ & $3.8 \%$ \\
\hline $\begin{array}{l}\text { El abuso sexual infantil en la familia disminuye a medida que aumenta } \\
\text { la edad del menor. }\end{array}$ & $30 \%$ & $48.8 \%$ & $15 \%$ & $6.3 \%$ \\
\hline
\end{tabular}

\section{Discusión}

El Abuso Sexual Infantil es una problemática que aflige a este país, en este caso, se sabe que las cifras de abuso sexual infantil en Mérida siguen incrementando, como se ha mencionado en el primer apartado. EI ASI es un hecho que ocurre tanto dentro como fuera de la familia, por eso es pertinente que quienes constituyen uno de los círculos sociales más cercanos a los y las menores de edad, en este caso, las y los profesores, se encuentren bien informados y capacitados para poder identificar y lidiar con este tipo de eventos, ya que son quienes pasan y conviven gran parte del día con ellos y ellas.

Con respecto al significado que los/las docentes le dan a este fenómeno social, se puede decir que tienen una connotación escasa ya que utilizan elementos como "el ASI es un tipo de violencia", "hay un contacto y acercamiento sexual", "no hay consentimiento del menor". Sin embargo, de acuerdo con otras definiciones, existen otros elementos importantes que deben conocer los docentes sobre la definición de este problema, entre los que se puede encontrar que, "estos contactos se dan entre un niño o niña y una persona mayor que él o ella" (Mendoza, 2009), "el agresor o la agresora usa al niño o niña para estimularse sexualmente a sí mismo, al niño o niña o a otra persona" (García, 2017), "este concepto tiene dos aspectos fundamentales: la coerción y la asimetría de edad, puesto que el agresor/a utiliza la situación de poder que tiene para relacionarse sexualmente con el o la menor y es mayor que la víctima" (Gil, 2012).

En lo relacionado con las opiniones que tienen acerca de este tema, las y los docentes entrevistados no mencionan que se dé más en un sexo que en otro, sino que plantean que se puede dar tanto en niños como en niñas, lo cual difiere con una investigación realizada por 
Giménez (2017), en donde se menciona que las que son mayormente afectadas son las niñas y que esta percepción se basa en la idea de fragilidad que se tiene sobre el sexo femenino en la cultura. Asimismo, esta investigación encontró que las consecuencias para los y las niñas víctimas de ASI no son solamente inmediatas, sino que también son perdurables y afectan gravemente el desarrollo y la calidad de vida de niños y niñas, por lo tanto, este es un punto que concuerda con las opiniones de las y los docentes entrevistados para esta investigación, y esto se puede observar en sus respuestas, donde mencionan que este problema afecta gravemente a niños y niñas en diferentes ámbitos de su vida.

Por otra parte, en lo que concierne al apartado de los factores de riesgo que pueden causar el abuso sexual infantil, se obtuvo que, muchos de los/las docentes piensan que en el factor familiar puede darse por tres razones principales: los padres y madres dejan a sus hijos o hijas al cuidado de algún familiar; los padres y madres pasan muchas horas fuera de casa por razones de trabajo; por último, puede influir la desestructuración familiar o el divorcio. Lo anterior coincide con la investigación de Redondo, Galdó y García (2008), en la cual se mencionan algunos factores de riesgo indirectos (en este caso, el cuidador/a), comenta que la presencia de un hombre o una mujer sin parentesco biológico podría dificultar o crear malos vínculos entre el encargado/a con el/la menor, incomprensión del cuidador/a al niño o niña, poca armonía en el matrimonio, métodos disciplinarios físicos fuertes, aislamiento social de la familia y pertenecer a familias numerosas.

Otro factor de riesgo del abuso sexual infantil es el contexto social; en este apartado muchos de los/las docentes piensan que se da por el consumo de drogas o sustancias alcohólicas, el bullying, debido a la globalización de la tecnología y la facilidad con la que los niños/niñas y los abusadores/as puede acceder a ellos mediante el uso de las redes sociales. Otro punto que expusieron fue la ignorancia y la falta de conciencia que la sociedad tiene ante esta situación que muchas veces se ve como normal. Estos factores muestran similitud con investigaciones como la de Rodondo, Galdó y García (2008), quienes mencionan el uso del alcohol y consumo de drogas como uno de los factores de riesgo. Sin embargo, no muestra resultados que mencionen la globalización de la tecnología o la ignorancia de la sociedad como factores de riesgo sociales que puedan intervenir en el abuso sexual infantil. Por otro lado, Sautu (2018) también menciona el alcohol y el uso de drogas como factores de riesgo, y por otra parte, en su investigación los resultados reflejaron también el factor de ser una víctima de discriminación de la sociedad y a los medios de comunicación como una vía para la violencia.

El último factor de riesgo que se trató fue el económico. La mayoría de los/las docentes mencionan que el nivel socioeconómico puede ser un factor o no, ya que, debido a la situación económica de estos tiempos, no les alcanza para vivir solo con el sueldo del padre y muchas veces la madre también tiene que trabajar, por consiguiente, dejar a un familiar al cuidado de sus hijos/as los hace más propensos a sufrir abuso sexual infantil. Esto se puede observar en la 
investigación realizada por Redondo, Galdó y García (2008) ya mencionada, en donde se observa que los profesores/as no piensan que el factor económico sea la causa directa para que se dé el ASI y que tampoco el ser una persona con estabilidad económica quiere decir que se sea menos propenso, ya que este problema afecta a todos los niveles socioeconómicos de la misma manera, aunque la mayoría de las veces son las personas con un nivel socioeconómico bajo en las que mayormente sucede esa situación o las que no cuentan con los suficientes recursos para defenderse.

En relación con la categoría de identificación del AFI, muchos de los/las docentes tienen conocimiento sobre indicadores que les hacen pensar que un niño/a está pasando por esa situación. De acuerdo con investigaciones realizadas por Mendoza (2009), los siguientes indicadores coinciden con las respuestas de las y los docentes: los niños/as dejan de asistir a clases y después lo hacen irregularmente, asisten sucios a la escuela o la ropa que llevan está rota, y pueden tener una conducta sexuada con sus compañeros/as. Lo anterior indica que los/las docentes poseen el conocimiento para identificar una posible situación de ASI, sin embargo, quedan muchos otros indicadores que no fueron mencionados. Acuña (2014), menciona que las víctimas tienen mayor tendencia a presentar depresión, ansiedad, trastornos de estrés postraumático, suicidio o ideación suicida, baja autoestima, enuresis o encopresis, trastornos alimenticios, trastornos del sueño, labilidad emocional, agresividad, desregulación emocional, conducta antisocial en la adultez temprana, esquizofrenia, bipolaridad, trastornos somatomorfos, déficit de atención e hiperactividad y problemas de memoria.

En cuanto a la categoría de "formas de actuar", la cual se refiere a las acciones que los/las docentes llevan a cabo al presentarse un caso de ASI, contestaron que lo primero que se hace es seguir el protocolo de actuación que poseen, por lo cual deben canalizar el caso con la autoridad inmediata, en este caso el director/a, quien se encargará de darle seguimiento dejándolos fuera, lo que refleja que sí saben cómo actuar, aunque no lo hacen directamente, y sí les gustaría hacerlo pero no lo tienen permitido. Este dato coincide con el resultado de los estudios de Mendoza (2009) y García (2017), que confirman que la mayoría de las profesoras/es saben qué hacer cuando se da un caso de abuso sexual en el contexto de la escuela y, aunque la forma de actuar varía dependiendo del país, se confirma que sí tienen conocimientos respecto a la forma correcta de actuar.

Por último, los datos obtenidos acerca de la experiencia de los/las docentes poseen en cuanto a casos de AFI, arrojaron que sí han tenido experiencias con al menos un caso dentro de su grupo o como mínimo han visto y/o escuchado de casos dentro de su medio de trabajo, lo cual coincide con datos de Bote (2019) acerca de que en Mérida, desde algunos años atrás ha habido un aumento considerable en las cifras reportadas de ASI; también coincide con lo mostrado en estudios donde se revela que han tenido la experiencia de vivir este hecho de cerca y debido a eso, poseen ciertos conocimientos que quizás otras personas no tan cercanas a los/las menores no pueden tener. 
Para terminar, esta investigación logró detectar la percepción social que tienen las y los docentes sobre el abuso sexual infantil. Se pudo notar qué significado tienen sobre este fenómeno, cómo se sienten al escuchar de estas situaciones y se descubrieron sus principales creencias. De igual manera, se describió cómo los/las docentes identifican posibles situaciones de ASI en sus alumnos/as, cuáles son los factores de riesgo principales y de qué manera deben actuar cuando se enteran de un caso de abuso sexual infantil.

También se pudo encontrar que les hace falta conocer más acerca del abuso sexual infantil porque, a pesar de tener el conocimiento sobre los protocolos de actuación, no se involucran directamente ya que todo se canaliza a la autoridad inmediata superior. Finalmente, se expone que los y las docentes tienen la percepción social de que el abuso sexual infantil es un problema que sí se da comúnmente hoy en día y que afecta a nuestro país.

\section{Referencias}

Acuña, M. (2014). Abuso sexual en menores de edad: generalidades, consecuencias y prevención. Medicina Legal de Costa Rica, 31(1).

http://www.scielo.sa.cr/scielo.php?script=sci arttext\&pid=S1409-00152014000100006

Bote, A. (2019). Abuso sexual infantil en Yucatán rebasa la media nacional. La Jornada Maya. Yucatán. https://www.lajornadamaya.mx/2019-04-30/Abuso-sexual-infantil-en-Yucatanrebasa-la-media-nacional

García, A. (2017). Evaluación psicosocial del abuso sexual infantil: factores de riesgo y protección, indicadores, técnicas y procedimientos de evaluación. Apuntes de Psicología, 34(2-3). http://apuntesdepsicologia.es/index.php/revista/article/view/611/457

Gil, A. (2012). Guía de recursos para la prevención y atención del abuso y la explotación sexual infantil. Madrid: Save the Children.

http://ibdigital.uib.es/greenstone/collect/cd2/archives/stc0085.dir/stc0085.pdf

Giménez, E. (2017). Percepción de los docentes sobre el abuso sexual en niños y niñas. Paraguay: UNAE. file://C:/Users/anapa/Downloads/8-45-2-PB.pdf

González, P. (2017). Referente de Pensamiento eje 3: Investigación cualitativa ¿Cómo la selección de la muestra en la población seleccionada es pertinente para contribuir a la metodología de investigación cualitativa? Areandina. Montevideo: Universidad de la República.

https://digitk.areandina.edu.co/handle/areandina/2575

Mendoza, B. (2009). Taller para la detección de casos de abuso sexual infantil en niños de educación básica. Psicología lberoamericana, 17(1). https://www.redalyc.org/pdf/1339/133912613004.pdf

Ortega, E.; B. Baz; F. Sánchez (2012). La conducta sexual infantil como indicador de abusos sexuales: los criterios y sesgos de los profesionales. Psicothema, 24(3).

https://www.redalyc.org/pdf/727/72723439010.pdf 
Priegue, D.; M. Cambeiro (2016). Los conocimientos acerca del maltrato infantil de los futuros profesionales de la educación: un estudio exploratorio. Revista Complutense de Educación, 27 (3). https://core.ac.uk/download/pdf/45655451.pdf

Redondo, C.; M. Galdó; M. García (2008). Atención al adolescente. España: Universidad de Cantabria. https://books.google.com.mx/books?id=uqJt5tN3sDYC\&pg=PA311\&lpg=PA311\&dq-v=0nepage \& $q \& \mathrm{f}=\mathrm{false}$

Sautu, R. (2018). La violencia y abuso de niños: testimonios docentes. Argentina: Universidad de Buenos Aires. http://lasa.international.pitt.edu/Lasa2000/Sautu.PDF

UNICEF (2016a). Abuso sexual contra niños, niñas y adolescentes: una guía para tomar acciones y proteger sus derechos. https://www.unicef.org/argentina/sites/unicef.org.argentina/files/2018-04/proteccion-AbusoSexual contra NNyA-2016.pdf UNICEF (2016b). Violencia contra los niños: nuevo informe oculto en plena luz. https://www.unicef.es/noticia/violencia-contra-los-ninos-nuevo-informe-ocultos-plena-luz 